\title{
Hetero-Cope Rearrangements of Nitrosobutenes. DFT studies of Thermal and Acid-Catalyzed Reactions
}

\author{
Arash Jabbari and K. N. Houk* \\ Department of Chemistry and Biochemistry, University of California, \\ Los Angeles, California 90095-1569 \\ houk@chem.ucla.edu
}

\section{SUPPORTING INFORMATIONS}

1) Full citation of Gaussian software ( reference 4).

2) Cartesian coordinates of all structures and calculated enthalphies (in Hartree).

1) Full citation of Gaussian software ( reference 4).

Gaussian 03, Revision C.02, Frisch, M. J.; Trucks, G. W.; Schlegel, H. B.; Scuseria, G. E.; Robb, M. A.; Cheeseman, J. R.; Montgomery, Jr., J. A.; Vreven, T.; Kudin, K. N.; Burant, J. C.; Millam, J. M.; Iyengar, S. S.; Tomasi, J.; Barone, V.; Mennucci, B.; Cossi, M.; Scalmani, G.; Rega, N.; Petersson, G. A.; Nakatsuji, H.; Hada, M.; Ehara, M.; Toyota, K.; Fukuda, R.; Hasegawa, J.; Ishida, M.; Nakajima, T.; Honda, Y.; Kitao, O.; Nakai, H.; Klene, M.; Li, X.; Knox, J. E.; Hratchian, H. P.; Cross, J. B.; Bakken, V.; Adamo, C.; Jaramillo, J.; Gomperts, R.; Stratmann, R. E.; Yazyev, O.; Austin, A. J.; Cammi, R.; Pomelli, C.; Ochterski, J. W.; Ayala, P. Y.; Morokuma, K.; Voth, G. A.; Salvador, P.; Dannenberg, J. J.; Zakrzewski, V. G.; Dapprich, S.; Daniels, A. D.; Strain, M. C.; Farkas, O.; Malick, D. K.; Rabuck, A. D.; Raghavachari, K.; Foresman, J. B.; Ortiz, J. V.; Cui, Q.; Baboul, A. G.; Clifford, S.; Cioslowski, J.; Stefanov, B. B.; Liu, G.; Liashenko, A.; Piskorz, P.; Komaromi, I.; Martin, R. L.; Fox, D. J.; Keith, T.; Al-Laham, M. A.; Peng, C. Y.; Nanayakkara, A.; Challacombe, M.; Gill, P. M. W.; Johnson, B.; Chen, W.; Wong, M. W.; Gonzalez, C.; and Pople, J. A.; Gaussian, Inc., Wallingford CT, 2004. 


\section{2) Cartesian coordinates of all structures and calculated enthalphies (in Hartree).}

$\begin{array}{lr}\text { 1, } & -668.974063 \\ \mathrm{C} & -2.157346 \\ \mathrm{C} & -0.922667 \\ \mathrm{C} & -1.894544 \\ \mathrm{C} & -2.656277 \\ \mathrm{C} & 0.139861 \\ \mathrm{C} & -0.415741 \\ \mathrm{H} & 0.176242 \\ \mathrm{H} & -0.340565 \\ \mathrm{H} & -2.326143 \\ \mathrm{H} & -0.515933 \\ \mathrm{C} & -1.896135 \\ \mathrm{H} & -1.309672 \\ \mathrm{H} & -2.918544 \\ \mathrm{C} & -1.294813 \\ \mathrm{H} & -0.409015 \\ \mathrm{H} & -2.018986 \\ \mathrm{H} & -3.539519 \\ \mathrm{H} & -2.581386 \\ \mathrm{C} & 1.497700 \\ \mathrm{O} & 1.787741 \\ \mathrm{O} & 2.367223 \\ \mathrm{C} & 3.699005 \\ \mathrm{H} & 4.259966 \\ \mathrm{H} & 3.682504 \\ \mathrm{H} & 4.139199 \\ \mathrm{~N} & 0.447827 \\ \mathrm{O} & 0.341636\end{array}$

$$
\begin{array}{rr}
0.567090 & -1.019733 \\
-0.303085 & -1.128797 \\
-0.132985 & 1.258023 \\
0.658857 & 0.218853 \\
0.302390 & -0.162327 \\
0.347740 & 1.270640 \\
-0.279765 & 1.944363 \\
1.377073 & 1.637596 \\
-0.027151 & 2.258390 \\
-0.347203 & -2.140596 \\
-1.623035 & 0.813641 \\
-2.214364 & 1.528087 \\
-2.013847 & 0.838079 \\
-1.725873 & -0.614912 \\
-2.365967 & -0.636625 \\
-2.158346 & -1.312694 \\
1.237771 & 0.475929 \\
1.060527 & -1.889730 \\
-0.391012 & -0.300951 \\
-1.219758 & -1.135457 \\
0.086708 & 0.613067 \\
-0.454335 & 0.544417 \\
0.054127 & 1.328530 \\
-1.533304 & 0.719109 \\
-0.258139 & -0.436434 \\
1.714319 & -0.768029
\end{array}
$$$$
-1.019733
$$$$
-0.132985 \quad 1.258023
$$$$
0.302390-0.162327
$$$$
-0.279765 \quad 1.944363
$$$$
-0.027151 \quad 2.258390
$$$$
-1.623035 \quad 0.813641
$$$$
-2.013847 \quad 0.838079
$$$$
-2.365967-0.636625
$$$$
1.237771 \quad 0.475929
$$$$
-0.391012-0.300951
$$$$
0.086708 \quad 0.613067
$$$$
0.054127 \quad 1.328530
$$$$
-0.258139-0.436434
$$$$
2.632280-0.003974
$$

TS10, -668.942920

$\begin{array}{lr}\mathrm{C} & 1.762404 \\ \mathrm{C} & 0.684113 \\ \mathrm{C} & 2.087474 \\ \mathrm{C} & 2.456635 \\ \mathrm{C} & -0.331319 \\ \mathrm{C} & 0.692842 \\ \mathrm{H} & 0.305961 \\ \mathrm{H} & 0.885726 \\ \mathrm{H} & 2.832964 \\ \mathrm{H} & -0.089265 \\ \mathrm{C} & 1.958877 \\ \mathrm{H} & 1.740961 \\ \mathrm{H} & 2.912558 \\ \mathrm{C} & 0.832954 \\ \mathrm{H} & -0.116129 \\ \mathrm{H} & 1.057911 \\ \mathrm{H} & 3.245767 \\ \mathrm{H} & 1.853660 \\ \mathrm{~N} & -0.243459 \\ \mathrm{O} & 0.864814 \\ \mathrm{C} & -1.641848 \\ \mathrm{O} & -1.882891 \\ \mathrm{O} & -2.541486 \\ & \end{array}$

$$
\begin{array}{rr}
0.485619 & 1.437081 \\
-0.415234 & 1.440105 \\
-0.346749 & -0.843823 \\
0.598189 & 0.253312 \\
0.521939 & -0.480175 \\
0.061142 & -1.472408 \\
-0.786166 & -2.042623 \\
0.903781 & -2.144387 \\
-0.334237 & -1.644388 \\
-0.308081 & 2.198103 \\
-1.766921 & -0.248163 \\
-2.484852 & -1.046094 \\
-2.060932 & 0.204607 \\
-1.777237 & 0.804476 \\
-2.105507 & 0.368656 \\
-2.490956 & 1.610768 \\
1.330531 & 0.121714 \\
1.247591 & 2.205131 \\
1.804840 & -0.006761 \\
2.340211 & -0.291496 \\
-0.160047 & -0.396456 \\
-1.226090 & -0.944451 \\
0.491427 & 0.370142
\end{array}
$$




\begin{tabular}{lr}
$\mathrm{C}$ & -3.832191 \\
$\mathrm{H}$ & -4.426807 \\
$\mathrm{H}$ & -4.286176 \\
$\mathrm{H}$ & -3.756068 \\
& \\
$\mathbf{2}$, & -669.002116 \\
$\mathrm{C}$ & 2.308527 \\
$\mathrm{C}$ & 2.783193 \\
$\mathrm{C}$ & 1.337644 \\
$\mathrm{C}$ & 1.791611 \\
$\mathrm{C}$ & -0.897439 \\
$\mathrm{C}$ & 0.008065 \\
$\mathrm{H}$ & 0.170215 \\
$\mathrm{H}$ & -0.504432 \\
$\mathrm{H}$ & 1.168319 \\
$\mathrm{H}$ & 3.157859 \\
$\mathrm{C}$ & 2.453284 \\
$\mathrm{H}$ & 2.155600 \\
$\mathrm{H}$ & 3.342077 \\
$\mathrm{C}$ & 2.822543 \\
$\mathrm{H}$ & 2.148608 \\
$\mathrm{H}$ & 3.822072 \\
$\mathrm{H}$ & 2.564122 \\
$\mathrm{H}$ & 2.302747 \\
$\mathrm{C}$ & -2.360432 \\
$\mathrm{O}$ & -3.184420 \\
$\mathrm{O}$ & -2.650148 \\
$\mathrm{C}$ & -4.025164 \\
$\mathrm{H}$ & -4.085974 \\
$\mathrm{H}$ & -4.323866 \\
$\mathrm{H}$ & -4.674059 \\
$\mathrm{~N}$ & -0.557231 \\
$\mathrm{O}$ & 0.750360 \\
& \\
\hline
\end{tabular}

$$
\begin{array}{rr}
-0.129368 & 0.454659 \\
0.526327 & 1.091541 \\
-0.216203 & -0.536320 \\
-1.127557 & 0.895417
\end{array}
$$

$$
\begin{array}{rr}
0.788554 & 1.023452 \\
-0.406706 & 1.387190 \\
-0.203640 & -1.080826 \\
1.069476 & -0.365665 \\
0.492495 & -0.170096 \\
-0.673628 & -0.474557 \\
-1.242964 & 0.448820 \\
-1.355412 & -1.160478 \\
0.043400 & -2.136144 \\
-0.547759 & 2.400265 \\
-1.260151 & -0.983630 \\
-2.168095 & -1.522480 \\
-0.870784 & -1.497384 \\
-1.605351 & 0.473214 \\
-2.378864 & 0.872188 \\
-2.060195 & 0.501156 \\
1.580078 & -0.954395 \\
1.627695 & 1.715498 \\
0.269727 & 0.092499 \\
1.116926 & 0.348447 \\
-1.055581 & 0.011719 \\
-1.389549 & 0.254165 \\
-2.473557 & 0.151495 \\
-1.079776 & 1.259224 \\
-0.896039 & -0.474296 \\
1.733568 & -0.116924 \\
2.088645 & -0.325100
\end{array}
$$

3, -286.386573

$\begin{array}{lr}\mathrm{C} & -2.214969 \\ \mathrm{C} & 0.900925 \\ \mathrm{C} & -0.591835 \\ \mathrm{C} & -1.456050 \\ \mathrm{H} & -2.257890 \\ \mathrm{H} & -2.822418 \\ \mathrm{H} & 1.081782 \\ \mathrm{H} & 1.460888 \\ \mathrm{H} & -0.909801 \\ \mathrm{H} & -0.705247 \\ \mathrm{H} & -1.437260 \\ \mathrm{O} & 1.947005 \\ \mathrm{~N} & 1.455068\end{array}$

$-0.846011$

$-0.201466$

$0.933297 \quad 0.165073$

1.079295

0.028751

$-0.187615$

0.458142

$-0.861264-1.288603$

$\begin{array}{ll}-1.581339 & 0.318942\end{array}$

$\begin{array}{ll}0.853791 & 1.243557\end{array}$

$\begin{array}{ll}1.782478 & -0.249353\end{array}$

$2.078888 \quad 0.140319$

$1.046555-1.277664$

$0.003300 \quad 1.548886$

$\begin{array}{ll}-1.076293 & 0.230598\end{array}$

$-0.269150 \quad-0.526524$

TS7, -286.340329

$\begin{array}{lrrr}\mathrm{C} & 1.364681 & -0.739685 & 0.220235 \\ \mathrm{C} & -1.414698 & 0.557842 & -0.244703 \\ \mathrm{C} & 0.365863 & 1.460282 & 0.269600 \\ \mathrm{C} & 1.263282 & 0.553562 & -0.302043 \\ \mathrm{H} & 1.227332 & -0.906748 & 1.283380 \\ \mathrm{H} & 1.966550 & -1.486621 & -0.287652\end{array}$




$\begin{array}{rrrr}\mathrm{H} & -1.274730 & 0.624151 & -1.324195 \\ \mathrm{H} & -2.169801 & 1.205208 & 0.200456 \\ \mathrm{H} & 0.221896 & 2.436689 & -0.189005 \\ \mathrm{H} & 0.197326 & 1.439261 & 1.342291 \\ \mathrm{H} & 1.578914 & 0.715006 & -1.330970 \\ \mathrm{O} & -0.358938 & -1.353878 & -0.268983 \\ \mathrm{~N} & -1.192964 & -0.598276 & 0.399860\end{array}$

TS8, -286.328936

$\begin{array}{lrrr}\mathrm{C} & 1.204695 & -0.993145 & 0.336278 \\ \mathrm{C} & -1.288281 & 0.846918 & 0.279912 \\ \mathrm{C} & 0.839322 & 1.369667 & 0.055387 \\ \mathrm{C} & 1.374254 & 0.167739 & -0.403090 \\ \mathrm{H} & 1.080450 & -0.944965 & 1.412556 \\ \mathrm{H} & 1.509580 & -1.955552 & -0.058944 \\ \mathrm{H} & -1.824187 & 1.724745 & -0.073189 \\ \mathrm{H} & -1.131994 & 0.737864 & 1.353011 \\ \mathrm{H} & 0.816092 & 2.237760 & -0.597556 \\ \mathrm{H} & 0.855599 & 1.595688 & 1.117034 \\ \mathrm{H} & 1.560830 & 0.063111 & -1.470218 \\ \mathrm{O} & -0.777804 & -1.272005 & 0.028849 \\ \mathrm{~N} & -1.346269 & -0.232812 & -0.503487\end{array}$

$\begin{array}{lrrr}\mathbf{4}, & -286.397270 & & \\ \mathrm{C} & 1.553142 & 0.050624 & 0.463149 \\ \mathrm{H} & 1.510820 & 0.160956 & 1.546705 \\ \mathrm{C} & 0.598418 & 0.912564 & -0.311430 \\ \mathrm{H} & 0.817246 & 1.975690 & -0.154229 \\ \mathrm{H} & 0.648700 & 0.696587 & -1.384523 \\ \mathrm{C} & 2.422308 & -0.790814 & -0.095000 \\ \mathrm{H} & 2.473522 & -0.924606 & -1.173646 \\ \mathrm{H} & 3.116145 & -1.378185 & 0.500373 \\ \mathrm{C} & -2.476956 & -0.595114 & 0.135424 \\ \mathrm{H} & -2.995474 & -1.491794 & -0.189826 \\ \mathrm{H} & -2.962984 & 0.099628 & 0.822037 \\ \mathrm{~N} & -1.303432 & -0.412855 & -0.326142 \\ \mathrm{O} & -0.758178 & 0.786018 & 0.145405\end{array}$

$\begin{array}{lrrr}\mathbf{5},-514.208766 & & \\ \mathrm{C} & 2.635937 & -0.588129 & 0.450311 \\ \mathrm{H} & 2.724669 & -0.541910 & 1.536837 \\ \mathrm{C} & 3.704577 & -0.327684 & -0.302376 \\ \mathrm{H} & 4.665810 & -0.078655 & 0.138432 \\ \mathrm{H} & 3.660105 & -0.356039 & -1.389143 \\ \mathrm{C} & 1.278702 & -0.957153 & -0.082169 \\ \mathrm{H} & 1.298872 & -1.028870 & -1.176029 \\ \mathrm{H} & 0.964591 & -1.939892 & 0.291168 \\ \mathrm{C} & 0.180975 & 0.042923 & 0.327449 \\ \mathrm{H} & 0.264316 & 0.337005 & 1.381538 \\ \mathrm{~N} & 0.351252 & 1.287062 & -0.517913 \\ \mathrm{O} & 0.729601 & 2.242252 & 0.115668 \\ \mathrm{C} & -1.218233 & -0.516144 & 0.071249 \\ \mathrm{O} & -1.479730 & -1.676703 & -0.150127 \\ \mathrm{O} & -2.130656 & 0.468863 & 0.146844 \\ \mathrm{C} & -3.497362 & 0.072659 & -0.076187 \\ \mathrm{H} & -3.810180 & -0.653468 & 0.678395\end{array}$




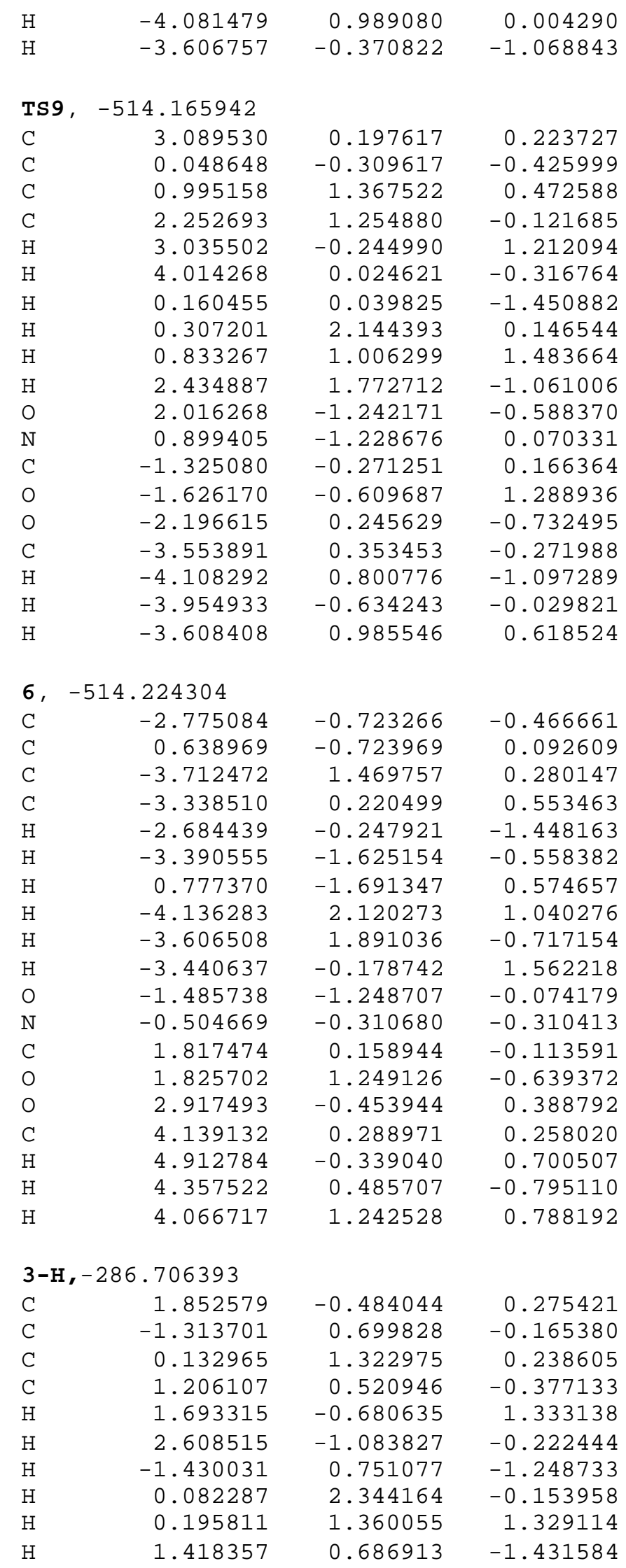




$\begin{array}{lrrr}\mathrm{O} & -0.440759 & -1.413191 & -0.329014 \\ \mathrm{~N} & -1.229281 & -0.668074 & 0.238511 \\ \mathrm{H} & -1.583165 & -0.962118 & 1.165912 \\ \mathrm{H} & -2.121749 & 1.208188 & 0.362004\end{array}$

$\begin{array}{lrrr}\text { TS7-H, } & -286.702646 & & \\ \mathrm{C} & 1.572492 & -0.030376 & 0.287505 \\ \mathrm{C} & -1.456926 & 0.032782 & -0.192050 \\ \mathrm{C} & -0.477038 & 1.355129 & 0.250215 \\ \mathrm{C} & 0.815156 & 1.021852 & -0.285613 \\ \mathrm{H} & 1.512244 & -0.224854 & 1.355710 \\ \mathrm{H} & 2.505347 & -0.331025 & -0.180291 \\ \mathrm{H} & -1.580454 & 0.085485 & -1.272457 \\ \mathrm{H} & -0.957427 & 2.223174 & -0.205547 \\ \mathrm{H} & -0.530278 & 1.391408 & 1.340212 \\ \mathrm{H} & 1.079100 & 1.355718 & -1.287338 \\ \mathrm{O} & 0.443101 & -1.216631 & -0.366994 \\ \mathrm{~N} & -0.730240 & -1.106047 & 0.189042 \\ \mathrm{H} & -0.776535 & -1.416920 & 1.164025 \\ \mathrm{H} & -2.407229 & 0.116073 & 0.338003\end{array}$

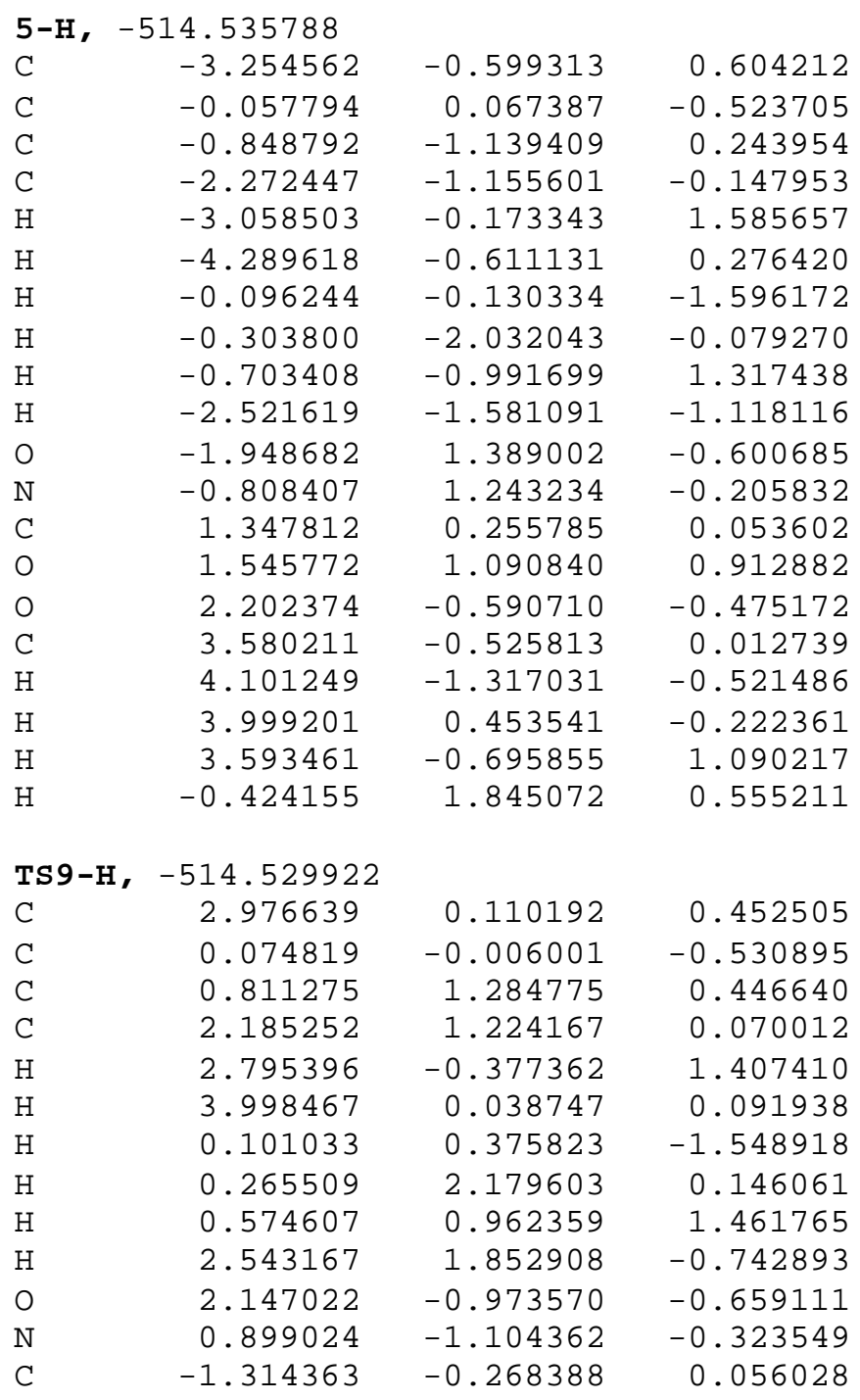




$\begin{array}{rrrr}\mathrm{O} & -1.453704 & -1.055927 & 0.966248 \\ \mathrm{O} & -2.229769 & 0.471973 & -0.534712 \\ \mathrm{C} & -3.601213 & 0.318220 & -0.054773 \\ \mathrm{H} & -4.179837 & 1.039089 & -0.628067 \\ \mathrm{H} & -3.938057 & -0.701506 & -0.247176 \\ \mathrm{H} & -3.641142 & 0.533618 & 1.014188 \\ \mathrm{H} & 0.684847 & -1.690344 & 0.494034\end{array}$

1-SbF5, $\mathrm{H}=-1173.636899$

$\begin{array}{lrrr}\text { C } & 2.025266 & -1.994724 & -0.309394 \\ \mathrm{C} & 1.781730 & -0.631860 & -0.919640 \\ \mathrm{C} & 3.737597 & -0.710023 & 0.773157 \\ \mathrm{C} & 3.034224 & -2.033028 & 0.569494 \\ \mathrm{C} & 1.483645 & 0.346551 & 0.287599 \\ \mathrm{C} & 2.697168 & 0.344388 & 1.248368 \\ \mathrm{H} & 3.158505 & 1.337753 & 1.258114 \\ \mathrm{H} & 2.356570 & 0.128008 & 2.263506 \\ \mathrm{H} & 4.545573 & -0.775753 & 1.507926 \\ \mathrm{H} & 0.945054 & -0.619091 & -1.614280 \\ \mathrm{C} & 4.276690 & -0.233736 & -0.603213 \\ \mathrm{H} & 4.738628 & 0.754501 & -0.489020 \\ \mathrm{H} & 5.058229 & -0.917626 & -0.948703 \\ \mathrm{C} & 3.100386 & -0.175619 & -1.612425 \\ \mathrm{H} & 2.979064 & 0.823226 & -2.031523 \\ \mathrm{H} & 3.264282 & -0.867243 & -2.444746 \\ \mathrm{H} & 3.342426 & -2.930434 & 1.098801 \\ \mathrm{H} & 1.398970 & -2.839188 & -0.576305 \\ \mathrm{C} & 1.111184 & 1.780550 & -0.137882 \\ \mathrm{O} & 1.623830 & 2.406190 & -1.032173 \\ \mathrm{O} & 0.177162 & 2.251701 & 0.699475 \\ \mathrm{C} & -0.417171 & 3.523917 & 0.363290 \\ \mathrm{H} & -1.223731 & 3.655733 & 1.081836 \\ \mathrm{H} & 0.326874 & 4.318875 & 0.448746 \\ \mathrm{H} & -0.808955 & 3.481506 & -0.654342 \\ \mathrm{~N} & 0.224334 & -0.196031 & 0.944782 \\ \mathrm{O} & 0.288963 & -0.627951 & 2.056050 \\ \mathrm{Sb} & -1.852620 & -0.384209 & -0.127356 \\ \mathrm{~F} & -1.014331 & -1.839923 & -0.932871 \\ \mathrm{~F} & -1.043116 & 0.722801 & -1.397472 \\ \mathrm{~F} & -2.357579 & 1.121734 & 0.828927 \\ \mathrm{~F} & -3.435657 & -0.575379 & -1.027369 \\ \mathrm{~F} & -2.246227 & -1.442725 & 1.333089\end{array}$

TS10-bF5, $\mathrm{H}=-1173.619827$

$\begin{array}{lrrr}\mathrm{C} & 1.930443 & -1.918811 & -0.655937 \\ \mathrm{C} & 1.849469 & -0.642049 & -1.274578 \\ \mathrm{C} & 3.570299 & -0.794843 & 0.764563 \\ \mathrm{C} & 2.783439 & -2.015444 & 0.406275 \\ \mathrm{C} & 1.337586 & 0.535852 & 0.616578 \\ \mathrm{C} & 2.622493 & 0.307631 & 1.375994 \\ \mathrm{H} & 3.184558 & 1.244578 & 1.417420 \\ \mathrm{H} & 2.354849 & 0.007808 & 2.393951 \\ \mathrm{H} & 4.337223 & -1.019970 & 1.511219 \\ \mathrm{H} & 0.973550 & -0.403450 & -1.869664 \\ \mathrm{C} & 4.218971 & -0.242496 & -0.525900 \\ \mathrm{H} & 4.809087 & 0.648725 & -0.287001 \\ \mathrm{H} & 4.909686 & -0.991630 & -0.927620\end{array}$




\begin{tabular}{|c|c|c|c|}
\hline C & 3.123638 & 0.106197 & -1.557932 \\
\hline $\mathrm{H}$ & 2.929238 & 1.180212 & -1.597446 \\
\hline $\mathrm{H}$ & 3.428785 & -0.189670 & -2.573051 \\
\hline $\mathrm{H}$ & 2.840122 & -2.915783 & 1.010940 \\
\hline $\mathrm{H}$ & 1.185864 & -2.676478 & -0.864867 \\
\hline $\mathrm{N}$ & 0.283855 & -0.279656 & 0.944920 \\
\hline 0 & 0.542807 & -1.273137 & 1.646105 \\
\hline C & 1.032512 & 1.935497 & 0.163728 \\
\hline 0 & 1.812581 & 2.633665 & -0.455988 \\
\hline 0 & -0.156265 & 2.328053 & 0.611514 \\
\hline C & -0.672123 & 3.573249 & 0.104095 \\
\hline $\mathrm{H}$ & -1.664391 & 3.659464 & 0.542503 \\
\hline $\mathrm{H}$ & -0.026976 & 4.401380 & 0.406081 \\
\hline $\mathrm{H}$ & -0.734765 & 3.524035 & -0.984972 \\
\hline $\mathrm{Sb}$ & -1.764459 & -0.388149 & -0.089286 \\
\hline $\mathrm{F}$ & -0.985048 & 0.725887 & -1.388760 \\
\hline $\mathrm{F}$ & -0.929647 & -1.824726 & -0.956316 \\
\hline $\mathrm{F}$ & -3.313789 & -0.640940 & -1.045938 \\
\hline $\mathrm{F}$ & -2.456170 & 1.077335 & 0.814518 \\
\hline $\mathrm{F}$ & -2.193099 & -1.481264 & 1.337309 \\
\hline 2-SbF5, & -1173.6646 & & \\
\hline $\mathrm{C}$ & -2.332483 & -1.521396 & 1.039883 \\
\hline C & -3.408537 & -1.011062 & 1.647383 \\
\hline C & -3.072795 & -0.508606 & -1.154191 \\
\hline C & -2.210656 & -1.566882 & -0.461112 \\
\hline C & -0.960449 & 0.852215 & -0.671129 \\
\hline C & -2.439747 & 0.880035 & -0.933117 \\
\hline $\mathrm{H}$ & -2.884583 & 1.404906 & -0.078816 \\
\hline $\mathrm{H}$ & -2.610189 & 1.528582 & -1.802789 \\
\hline $\mathrm{H}$ & -3.074923 & -0.724650 & -2.228029 \\
\hline $\mathrm{H}$ & -3.454488 & -1.011662 & 2.735191 \\
\hline C & -4.510829 & -0.598949 & -0.609780 \\
\hline $\mathrm{H}$ & -5.152205 & 0.132677 & -1.115868 \\
\hline $\mathrm{H}$ & -4.907091 & -1.589169 & -0.868254 \\
\hline C & -4.581100 & -0.404988 & 0.918499 \\
\hline $\mathrm{H}$ & -4.640713 & 0.664406 & 1.174460 \\
\hline $\mathrm{H}$ & -5.517567 & -0.834876 & 1.298129 \\
\hline $\mathrm{H}$ & -2.445018 & -2.565606 & -0.846081 \\
\hline $\mathrm{H}$ & -1.495073 & -1.930354 & 1.598484 \\
\hline C & -0.274530 & 2.193111 & -0.482724 \\
\hline O & 0.430076 & 2.692310 & -1.322615 \\
\hline 0 & -0.678071 & 2.749661 & 0.662402 \\
\hline C & -0.017617 & 3.987610 & 1.006638 \\
\hline $\mathrm{H}$ & -0.481565 & 4.312022 & 1.937346 \\
\hline $\mathrm{H}$ & 1.047525 & 3.794170 & 1.149021 \\
\hline $\mathrm{H}$ & -0.161199 & 4.728741 & 0.217210 \\
\hline $\mathrm{N}$ & -0.247304 & -0.213037 & -0.633949 \\
\hline 0 & -0.821531 & -1.446612 & -0.873369 \\
\hline $\mathrm{F}$ & 1.852294 & -2.045664 & -0.984533 \\
\hline $\mathrm{F}$ & 0.984324 & -1.460828 & 1.422436 \\
\hline $\mathrm{F}$ & 3.554828 & -0.795856 & 0.772793 \\
\hline $\mathrm{F}$ & 1.556960 & 1.044753 & 1.025802 \\
\hline $\mathrm{Sb}$ & 1.877276 & -0.534573 & 0.076919 \\
\hline $\mathrm{F}$ & 2.404463 & 0.488253 & -1.368576 \\
\hline
\end{tabular}




\begin{tabular}{|c|c|c|c|}
\hline \multicolumn{4}{|c|}{ 1-TiCl4, -2568.079273} \\
\hline $\mathrm{C}$ & -2.687263 & -1.938582 & 0.758575 \\
\hline $\mathrm{C}$ & -2.123906 & -0.577903 & 1.077136 \\
\hline $\mathrm{C}$ & -3.776101 & -0.610739 & -0.911921 \\
\hline $\mathrm{C}$ & -3.565876 & -1.952695 & -0.252514 \\
\hline $\mathrm{C}$ & -1.388890 & -0.032749 & -0.249478 \\
\hline $\mathrm{C}$ & -2.396387 & -0.105065 & -1.424162 \\
\hline $\mathrm{H}$ & -2.499290 & 0.877840 & -1.891703 \\
\hline $\mathrm{H}$ & -2.000453 & -0.796844 & -2.172586 \\
\hline $\mathrm{H}$ & -4.471908 & -0.665516 & -1.754135 \\
\hline $\mathrm{H}$ & -1.408052 & -0.591226 & 1.899229 \\
\hline $\mathrm{C}$ & -4.287750 & 0.389505 & 0.160557 \\
\hline $\mathrm{H}$ & -4.336450 & 1.393322 & -0.276294 \\
\hline $\mathrm{H}$ & -5.303171 & 0.118894 & 0.465469 \\
\hline $\mathrm{C}$ & -3.322707 & 0.366519 & 1.375529 \\
\hline $\mathrm{H}$ & -2.978659 & 1.372059 & 1.631159 \\
\hline $\mathrm{H}$ & -3.820244 & -0.032201 & 2.264501 \\
\hline $\mathrm{H}$ & -4.068125 & -2.849612 & -0.603391 \\
\hline $\mathrm{H}$ & -2.384996 & -2.812103 & 1.327285 \\
\hline $\mathrm{C}$ & -0.770341 & 1.324824 & 0.022930 \\
\hline 0 & 0.392701 & 1.448175 & 0.405496 \\
\hline 0 & -1.566032 & 2.359962 & -0.154558 \\
\hline $\mathrm{C}$ & -0.986369 & 3.671923 & 0.085540 \\
\hline $\mathrm{H}$ & -0.678160 & 3.754267 & 1.129332 \\
\hline H & -0.125306 & 3.816068 & -0.568697 \\
\hline $\mathrm{H}$ & -1.783681 & 4.375615 & -0.146894 \\
\hline $\mathrm{N}$ & -0.174397 & -0.920130 & -0.457810 \\
\hline o & -0.390157 & -1.991483 & -0.949220 \\
\hline $\mathrm{Ti}$ & 1.917198 & -0.140176 & 0.058147 \\
\hline $\mathrm{Cl}$ & 3.497625 & 1.287963 & 0.603299 \\
\hline $\mathrm{Cl}$ & 1.567165 & 0.55 & -2 \\
\hline $\mathrm{Cl}$ & 3.001699 & -1.967079 & -0.482826 \\
\hline $\mathrm{Cl}$ & 1.300709 & -0.831694 & 2.145189 \\
\hline & C14, -256 & 59574 & \\
\hline $\mathrm{C}$ & -2.368190 & -1.836662 & 0.976581 \\
\hline $\mathrm{C}$ & -2.281875 & -0.488346 & 1.390201 \\
\hline C & -3.586523 & -0.876975 & -0.917014 \\
\hline $\mathrm{C}$ & -3.005552 & -2.062341 & -0.215803 \\
\hline $\mathrm{C}$ & -1.253420 & 0.22 & -0.589339 \\
\hline $\mathrm{C}$ & -2.434036 & 0.043126 & -1.497976 \\
\hline $\mathrm{H}$ & -2.868846 & 1.013595 & -1.750634 \\
\hline $\mathrm{H}$ & -2.062218 & -0.425657 & -2.414753 \\
\hline $\mathrm{H}$ & -4.217755 & -1.182595 & -1.755766 \\
\hline $\mathrm{H}$ & -1.526990 & -0.219318 & 2.123706 \\
\hline $\mathrm{C}$ & -4.404372 & -0.058157 & 0.105658 \\
\hline $\mathrm{H}$ & -4.867859 & 0.799360 & -0.393508 \\
\hline $\mathrm{H}$ & -5.214224 & -0.678650 & 0.503421 \\
\hline $\mathrm{C}$ & -3.476220 & 0.416784 & 1.242532 \\
\hline $\mathrm{H}$ & -3.156318 & 1.453284 & 1.102589 \\
\hline $\mathrm{H}$ & -3.998307 & 0.397782 & 2.210854 \\
\hline $\mathrm{H}$ & -3.033208 & -3.048556 & -0.668307 \\
\hline $\mathrm{H}$ & -1.755708 & -2.596962 & 1.450294 \\
\hline $\mathrm{N}$ & -0.265545 & -0.735007 & -0.638792 \\
\hline 0 & -0.590995 & -1.815094 & -1.148200 \\
\hline C & -0.683661 & 1.497889 & -0.148049 \\
\hline 0 & 0.476107 & 1.577896 & 0.27588 \\
\hline
\end{tabular}




$\begin{array}{lrrr}\mathrm{O} & -1.488284 & 2.553668 & -0.216481 \\ \mathrm{C} & -0.910028 & 3.822726 & 0.182959 \\ \mathrm{H} & -1.705580 & 4.552257 & 0.038515 \\ \mathrm{H} & -0.599463 & 3.780230 & 1.228776 \\ \mathrm{H} & -0.047961 & 4.050257 & -0.446386 \\ \mathrm{Ti} & 1.845742 & -0.170970 & 0.054430 \\ \mathrm{Cl} & 1.010052 & -0.757006 & 2.130897 \\ \mathrm{Cl} & 3.515521 & 1.074588 & 0.787157 \\ \mathrm{Cl} & 1.872129 & 0.543588 & -2.127492 \\ \mathrm{Cl} & 2.752625 & -2.133199 & -0.344847\end{array}$

\begin{tabular}{|c|c|c|c|}
\hline $\mathrm{C}$ & -3011216 & -1 & \\
\hline $\mathrm{C}$ & -4.136050 & -1.066937 & $\begin{array}{l}.084408 \\
1.375982\end{array}$ \\
\hline C & -3.136304 & -0.056886 & -1.114565 \\
\hline C & -2.566327 & -1.353598 & -0.534153 \\
\hline $\mathrm{C}$ & -0.979691 & 0.871075 & -0.256508 \\
\hline $\mathrm{C}$ & -2.436723 & 1.149386 & -0.463315 \\
\hline $\mathrm{H}$ & -2.883467 & 1.396631 & 0.507297 \\
\hline $\mathrm{H}$ & -2.544489 & 2.042958 & -1.088112 \\
\hline $\mathrm{H}$ & -2.923590 & -0.040930 & -2.189752 \\
\hline $\mathrm{H}$ & -4.423520 & -1.289524 & 2.402146 \\
\hline $\mathrm{C}$ & -4.660955 & -0.051472 & -0.895042 \\
\hline $\mathrm{H}$ & -5.096852 & 0.851445 & -1.338951 \\
\hline $\mathrm{H}$ & -5.087351 & -0.902689 & -1.440649 \\
\hline $\mathrm{C}$ & -5.048133 & -0.155900 & 0.593653 \\
\hline $\mathrm{H}$ & -5.049730 & 0.837608 & 1.067936 \\
\hline $\mathrm{H}$ & -6.084417 & -0.508832 & 0.678371 \\
\hline $\mathrm{H}$ & -2.822094 & -2.205267 & -1.173850 \\
\hline $\mathrm{H}$ & -2.385160 & -2.256369 & 1.480129 \\
\hline $\mathrm{C}$ & 0.015996 & 1.918963 & 0.061346 \\
\hline 0 & 1.207961 & 1.656658 & 0.231198 \\
\hline 0 & -0.481320 & 3.138953 & 0.148536 \\
\hline $\mathrm{C}$ & 0.462323 & 4.196174 & 0.459438 \\
\hline $\mathrm{H}$ & -0.134049 & 5.106522 & 0.488768 \\
\hline $\mathrm{H}$ & 0.931308 & 3.999372 & 1.425371 \\
\hline $\mathrm{H}$ & 1.226080 & 4.248778 & -0.318788 \\
\hline $\mathrm{N}$ & -0.408254 & -0.283615 & -0.337758 \\
\hline 0 & -1.100616 & -1.401594 & -0.610594 \\
\hline $\mathrm{Ti}$ & 1.914380 & -0.441565 & 0.020649 \\
\hline $\mathrm{Cl}$ & 1.923530 & 0.138361 & -2.191681 \\
\hline $\mathrm{Cl}$ & 1.980881 & -2.613912 & -0.313719 \\
\hline $\mathrm{Cl}$ & 1.160109 & -0.564210 & 2.18255 \\
\hline $\mathrm{Cl}$ & 3.993893 & 0.107113 & 0.47516 \\
\hline
\end{tabular}

TiCL4, $\quad-1899.098619$

$\begin{array}{lrrr}\text { Ti } & 0.000000 & 0.000000 & 0.000000 \\ \mathrm{Cl} & 1.249363 & 1.249363 & 1.249363 \\ \mathrm{Cl} & -1.249363 & -1.249363 & 1.249363 \\ \mathrm{Cl} & 1.249363 & -1.249363 & -1.249363 \\ \mathrm{Cl} & -1.249363 & 1.249363 & -1.249363\end{array}$

$\begin{array}{lrrr}\text { SbF5, } & -504.631374 & & \\ \text { Sb } & 0.000000 & 0.000000 & 0.000000 \\ \text { F } & 0.000000 & 0.000000 & 1.839534 \\ \text { F } & 0.000000 & 1.825151 & 0.000000 \\ \text { F } & -1.580627 & -0.912575 & 0.000000\end{array}$


0.000000

$-1.839534$

$1.580627-0.912575$

0.000000 